\title{
Free-field evoked auditory brainstem responses in cochlear implant users
}

\author{
Erik Schebsdat, ${ }^{1}$ Manuel C. Kohl, ${ }^{1}$ Farah I. Corona-Strauss, ${ }^{1,2}$ Harald Seidler, ${ }^{3}$ \\ Daniel J. Strauss ${ }^{1,2}$ \\ ${ }^{1}$ Systems Neuroscience \& Neurotechnology Unit, Saarland University, Faculty of Medicine, Homburg/Saar \\ and Saarland University of Applied Sciences, Saarbruecken; ${ }^{2}$ Key Numerics GmbH, Saarbruecken; \\ ${ }^{3}$ MediClin Bosenberg Kliniken, St. Wendel, Germany
}

\begin{abstract}
The importance of binaural cues in auditory stream formation and sound source segregation is widely accepted. When treating one ear with a cochlear implant (CI) the peripheral auditory system gets partially replaced and processing delays get added potentially, thus important interaural time differences get altered. However, these effects are not fully understood, leaving a lack of systematic binaural fitting strategies with respect to an optimal binaural fusion. To get new insights into such alterations, we suggest a novel method of free-field auditory brainstem evoked responses (ABRs) analysis in CI users. This method does not bypass the technically induced intrinsic delays of the sound processor while leaving the whole electrode array active, thus the most natural way of stimulation is provided. We compared the ABRs collected of $12 \mathrm{CI}$ users and 12 normal hearing listeners using two different stimuli (chirp, click) at four different intensities each. We analyzed the ABRs using the average of 2000 trials
\end{abstract}

Correspondence: Daniel J. Strauss, Systems Neuroscience \& Neurotechnology Unit, Saarland University, Faculty of Medicine, Homburg/Saar, Germany.

E-mail: daniel.strauss@uni-saarland.de

Key words: Cochlear implants; Sensorineural hearing loss; Neurology; Auditory brainstem response; Interaural-time-difference.

Contributions: ES: measurement design, data collecting, analyzing, manuscript writing, references research; MCK: data analyzing, manuscript reviewing; FICS: measurement design, manuscript reviewing; HS: Head of department (MediClin Bosenberg Kliniken, St.Wendel, Germany), supervising patient measurements; DJS: supervisor.

Conflict of interest: the authors declare no potential conflict of interest.

Funding: this study was partially supported by the German Federal Ministry of Education and Research (Grant-no.: BMBF-FZ 03FH016PX5).

Received for publication: 5 September 2018.

Accepted for publication: 31 October 2018.

This work is licensed under a Creative Commons Attribution NonCommercial 4.0 License (CC BY-NC 4.0).

(C) Copyright E. Schebsdat et al., 2018

Licensee PAGEPress, Italy

Audiology Research 2018;8:216

doi:10.4081/audiores.2018.216 as well as a single trial analysis and found consistent results in the ABRs' amplitudes and latencies, as well as in single trial relationships between both groups. This method provides a new perspective into the natural CI users' ABRs and can be useful in future research regarding binaural interaction and fusion.

\section{Introduction}

The cochlear implant (CI) is the only device that can restore partial hearing to a totally deafened person via electric stimulation of the residual auditory nerve. ${ }^{1}$ Today, it is a complex multielectrode device that supports most of its approximately 320,000 users in daily-life situations. During recent years, the implant candidacy criteria have been altered in order to include young children and adults who have significant functional residual hearing, particularly at low frequencies, as well as people with single-sided deafness. ${ }^{1}$ Besides, note that the importance of binaural cues in exogenously driven auditory stream formation and sound source segregation is widely accepted. ${ }^{2,3}$ They may also reduce listening effort and fatigue. ${ }^{3}$ The CI treatment mediates such binaural processing to some extent. However, it therefore partially replaces the peripheral auditory system and might also add processing delays, thus important interaural time differences (ITD) get altered. These effects are far from being understood and systematic binaural fitting strategies with respect to an optimal binaural fusion are still missing. This seems to be a substantial lack of knowledge and techniques, especially when taking recent neurobiological and neuromodeling research into account. Those emphasize the necessity of a high spatiotemporal precision of neural coincidence detection circuit activations to mediate binaural interaction. ${ }^{4-6}$ Accordingly, there is a need for a robust method that provides objective insights into the neural processing responses of CI users that is suitable for use in diagnostics and research. Such insights could be used to enhance the CI fitting with respect to the users' individual neural behavior and furthermore offer a general comparison between the neural reaction of CI users and that of normal hearing listeners $(\mathrm{NH})$ - and perhaps an alignment of the neural responses in the future.

A well-established technique in audiology that offers insights into the integrity of the residual auditory nerve is the measurement of auditory evoked responses (for our interest the brainstem evoked response audiometry). ${ }^{7}$ This method consists of multiple acquisitions of auditory brainstem responses (ABRs) to different intensity levels and is used as a preliminary investigation prior a CI implantation. ${ }^{1}$ There are several studies that aim for an equivalent procedure in CI users by using a pure electric (internal) stimulation of a single, isolated implant electrode, like electrically evoked auditory brainstem responses (eABR), ${ }^{8-10}$ or by using the 
direct-audio-in channel to supply external stimulations. ${ }^{11}$ However, all these studies have in common that they do not take essential elements of the CI into account, for instance the microphone, the digital sound processor with its filter bank, and other processing parts of the device. Besides, eABR measurements stimulate only with a single isolated implant electrode (and not with the complete electrode array like in CI users natural listening situations), thus a comparison between NH listeners' and CIs' true/natural evoked response patterns and their temporal appearance is rather infeasible. Note that such insights into the true temporal appearance of the neural responses and furthermore into the ITDs could also help in balancing all kinds of CI configurations: unilateral, bilateral and bimodal. ${ }^{12,13}$

Another problem that arises when investigating auditory evoked potentials in CI users is the prominent CI artifact caused by the electrical stimulation, which can overlap the desired neural response. ${ }^{14}$ Recently, some methods have been developed which promise to remove the artifact from single channel ${ }^{15,16}$ and from multichannel cortical auditory evoked responses. ${ }^{17,18}$ However, these methods only concentrate on cortical potentials (and not ABRs) and they are limited in the evaluation since they lack the actual (artifact free) responses for comparison. ${ }^{14}$ Besides artifact removal in cortical potentials, there are subtraction techniques to obtain the small neural response from eABRs. ${ }^{19-21}$ However, as previously mentioned, eABR measurements are based on single electrode stimulations, preventing an adaptation of this subtraction method to eliminate artifacts caused by whole-array stimulations.

Therefore, it is the aim of this study to introduce a novel method that offers the interpretation of narrowband filtered neural correlates of free-field evoked ABRs, using different kinds of stimuli and intensities. All intrinsic delays and implant electrodes are included, thereby offering insights into the true-to-life temporal appearance of the CI users' responses. With this method, we found consistent differences in the ABRs' amplitudes and latencies, as well as in single trial relationships between NHs and CI users.

\section{Materials and Methods}

\section{Participants}

The study was performed in accordance with the declaration of Helsinki and was approved as a scientific study by the local ethics committee (Aerztekammer des Saarlandes; Medical Council of the Saarland, Germany). All participants provided written consent form after a detailed explanation. This study was conducted at the MediClin Bosenberg clinic, St.Wendel, Germany and at the Saarland University of Applied Sciences (htwsaar), Saarbruecken, Germany, and was performed on a control-group of $12 \mathrm{NHs}(\varnothing$ hearing threshold $\leq 15 \mathrm{~dB}$ hearing level (HL); $7 \mathrm{f} / 5 \mathrm{~m}$; $\varnothing$ age approximately $27.92 \pm 8.7 \mathrm{y}$ ) and a patient-group (Table 1 ) of $12 \mathrm{CI}$ users ( $\varnothing$ hearing threshold $\leq 60 \mathrm{~dB}$ HL; $9 \mathrm{f} / 3 \mathrm{~m}$; $\varnothing$ age $49.83 \pm 13.9$ y) with no further neurological disorders.

\section{Experimental setup}

The measurements were conducted in an acoustically shielded room using an audiometric free-field configuration. Each participant sat on a comfortable chair in front of a loudspeaker (Control $1 G$ Universe JBL, $1.0 \mathrm{~m}$ distance; Renkforce audio amplifier SAP702) and was asked to relax and keep eyes closed during the procedure. For the CI group all additional filters of the sound processor like noise blocks, wind blocks and gain controls were turned off and an omnidirectional microphone characteristic was used. In bilateral and bimodal users the contralateral device (CI or hearing aid) was turned off. Two stimuli, the A-chirp by Fobel and Dau ${ }^{22}$ (edge frequencies $10-10,000 \mathrm{~Hz}$, total length of $10.17 \mathrm{~ms}$, inter stimulus interval of $60 \mathrm{~ms}$ ) and an alternating-polarity click $^{7}$ (total length of $100 \mu \mathrm{s}$, inter stimulus interval of $66.0 \mathrm{~ms}$ ) were used. Each stimulus was presented at four intensities: 40, 50, 60, and 70 $\mathrm{dB}$ peak equivalent sound pressure level (peSPL). Thus, a total of 8 measurements were recorded with 3000 trials each (total measurement length of approximately 25 minutes) for each subject. We used a laptop with the digital audio workstation PreSonus Studio One 2 (2.5.0) and the Scarlett 2i4 USB audio interface to present the stimulus. The audio interface additionally offered the trigger signal.

\section{Data acquisition}

Four passive $\mathrm{Ag} / \mathrm{AgCl}$-electrodes were placed in total at the left and right mastoid, the reference at the vertex $\left(\mathrm{C}_{\mathrm{Z}}\right)$ and ground at the forehead. Impedances were kept below $5 \mathrm{k} \Omega$. ABRs were recorded using a commercially available biosignal amplifier (g.USBamp, Guger Technologies, Austria) with a sampling rate of $19,200 \mathrm{~Hz}$. To format the trigger signal of the auditory stimulation, a trigger conditioner (g.TRIGbox, Guger Technologies, Austria) was used. First offline analysis was performed using MATLAB

Table 1. Overview of the 12 participated cochlear implant (CI) users with the equipped sound processor, implant, CI-configuration (ma, monaural; ba, binaural), and the individual CI-experience in months.

\begin{tabular}{lllll} 
Participant & Sound processor & Implant & CI-configuration/used side & CI-experience [months] \\
1 & CP910 & CI422 & ba (right) & 11 \\
2 & Naida Q70 & HR90K Advantage (HiFocus ms) & ba (right) & 26 \\
\hline 3 & Sonnet & Synchrony (Medium) & ma (right) & 12 \\
4 & CP910 & CI422 & ma (right) & 6 \\
\hline 5 & CP910 & CI422 & ba (right) & 50 \\
6 & Sonnet & Synchrony (Flex28) & ba (right) & 7 \\
\hline 7 & CP910 & CI512 & ma (left) & 26 \\
8 & Naida Q70 & HR90K Advantage (HiFocus ms) & 15 \\
\hline 9 & CP910 & CI24RE & ba (left) & 78 \\
10 & Sonnet & SonatarTi100 (FlexSoft) & ba (left) & 63 \\
\hline 11 & CP910 & CI512 & ba (right) & 14 \\
12 & CP910 & CI522 & & 20 \\
\hline
\end{tabular}


(9.0 R2016a, 64Bit) by separately applying two zero-phase FIR1 bandpass-filters of $150-1500 \mathrm{~Hz}$ (conventional ABR frequency band) ${ }^{7}$ and of $150-300 \mathrm{~Hz}$ (narrow frequency band), the latter was done in order to attenuate the $\mathrm{CI}$ artifact. ${ }^{14-16}$ Additionally a $50 \mathrm{~Hz}$ zero-phase IIR notch (notches at all integer multiples of $50 \mathrm{~Hz}$ ) was used in both cases. The artifact filter was set to $25.0 \mu \mathrm{V}$ yielding a total of 2000 used artifact-free trials.

\section{Wavelet phase synchronization stability}

The wavelet phase synchronization stability (WPSS) reflects the phase stability between the single evoked trials in a specific frequency range and allows us to exclude noisy frequencies. ${ }^{23,24} \mathrm{By}$ applying the continuous wavelet transform with a complex mother wavelet, the sixth derivative of the complex Gaussian function in this case, to the ABR signals, the instantaneous phase can be extracted. This wavelet function $\psi \in L^{2}(\mathbb{R})$ ( $L^{2}$ denoting the Hilbert space of square integrable functions, i.e., all functions $x$ that satisfy $\|x(t)\|_{L^{2}}^{2}<\infty$ ) and satisfies the admissibility criterion:

$$
0<C_{\psi}=\int_{\mathbb{R}} \frac{|\Psi(\omega)|^{2}}{|\omega|} d \omega<\infty
$$

$C_{\psi}$ is the admissibility constant and $\Psi(\omega)$ is the Fourier transform of the wavelet $\psi$. By stretching or compressing the mother wavelet $\psi \in L^{2}(\mathbb{R})$ corresponding to the scale parameter $a$ and shifting it in time according to the parameter $b$, a set of wavelets $\psi_{a, b}$ is obtained: 25

$$
\psi_{a, b}(\cdot)=|a|^{-\frac{1}{2}} \psi\left(\frac{-b}{a}\right), \quad a, b \in \mathbb{R}, a \neq 0
$$

Using these, we are able to analyze signals simultaneously in time and scale. The wavelet transform $\mathrm{W}_{\psi}: L^{2} \rightarrow L^{2}\left(\mathbb{R}, \frac{d a d b}{a^{2}}\right)$ of a signal $\psi \in L^{2}(\mathbb{R})$ is then given by the inner $L^{2}$ - product: ${ }^{26}$

$$
\begin{aligned}
\left(W_{\psi} x\right)(a, b) & =\left\langle x, \psi_{a, b}\right\rangle_{L^{2}} \\
& =\int_{\mathbb{R}} x(t) \psi_{a, b}^{*}(t) d t
\end{aligned}
$$

Note that the scale parameter can always be associated with a so-called pseudofrequency $f_{a}$ by

$$
f_{a}=\frac{f_{\psi}}{a}
$$

where $f_{\psi}$ is the center frequency of the wavelet in Hz. When using a complex wavelet, the instantaneous phase $\varphi$ of a signal $x$ can be extracted by

$$
\varphi_{a, b}(x)=\arg \left(\left(W_{\psi} x\right)(a, b)\right)
$$

Now, the WPSS $\Gamma_{a, b}$ of an ABR sequence $X=\left\{x_{m} \in L^{2}(\mathbb{R}): m\right.$ $=1, \ldots, M\}$ of $M$ sweeps is defined by

$$
\Gamma_{a, b}(X):=\frac{1}{M}\left|\sum_{m=1}^{M} e^{i \varphi_{a, b}\left(x_{m}\right)}\right|
$$

and assumes values between 0 and 1. Equal phase dispersion across single trials is represented by 0 , whereas a perfect synchro- nization is represented by 1 . In this study, we used scales ranging from 40-75 (step size of 5) reflecting a pseudofrequency range of $154-288 \mathrm{~Hz}$ (fullscale WPSS).

\section{Estimation of the free-field evoked auditory brainstem evoked response latencies}

Since we present a novel method of measuring and analyzing free-field evoked ABR correlates, we need to estimate the temporal occurrence of the responses, more precisely, those of the prominent wave $\mathrm{V}\left(W_{V}\right)$. We calculated the temporal position of $W_{V}$ for the stimuli as:

$$
\begin{gathered}
t_{W_{V}}=\text { Stimulus Traveling Time } \\
+ \text { Neural delay of } W_{V} \\
+ \text { Stimulus Length }
\end{gathered}
$$

The stimulus traveling time was calculated using the sound velocity $\left(343 \frac{\mathrm{m}}{\mathrm{s}}\right.$ ) and the distance between the loudspeaker and the participant $(1 \mathrm{~m})$, and determined to be approximately $3 \mathrm{~ms}$. For the neural delay of the $W_{V}$ we used the constant of $5 \mathrm{~ms}$ from the literature. ${ }^{7,22}$ According to Equation 7, this results in the following estimations:

$$
\begin{array}{ll}
t_{W_{V}-\text { chirp }} & \approx 18 \mathrm{~ms}, \\
t_{W_{V}-\text { click }} & \approx 8 \mathrm{~ms} .
\end{array}
$$

\section{Narrowband filtering, oscillations and ringing artifact}

One major issue when investigating evoked potentials in CI users is the prominent CI artifact ${ }^{14-18}$ that can overlap the desired response pattern, making the interpretation difficult or even impossible especially when considering conventional artifact filtering. Consequently, Luu and Tucker ${ }^{27}$ suggested that bandpass filtering around the peak frequency of the component of interest (in our case the $W_{V}$ ) can be used to remove the contribution of other superimposed components (in our case the CI artifact), thus uncovering the true underlying oscillations. According to Yeung et al. ${ }^{28}$ the exogenously driven synchronization of these oscillations should produce a series of peaks of diminishing amplitude and result in the well-known ABR pattern in the average response. However, both Luu and Tucker ${ }^{27}$ and Yeung et al. ${ }^{28}$ demonstrated the downside of narrowband filtering evoked potentials because this technique smears out parts of the signal in time due to the narrow filter bandwidth, causing a distortion, the so called ringing artifact. This artifact may create the appearance of oscillating activity where none is present. Although the magnitude of the ringing artifact will depend on the size of the original peak in the average pattern, the appearance of oscillations in narrowband filtered data is not definitely evidence of synchronized oscillations (the desired responses). In other words, applying this technique helps to filter out unwanted superimposed components like the CI artifact, but in turn induces unwanted oscillations, which pose the threat of misinterpreting them as actual neural activity. Figure 1 shows our adaption of the work of Yeung et al. ${ }^{28}$ by presenting our grand average $\mathrm{NH}$ chirp- and click-stimulus responses $(60 \mathrm{~dB}$ peSPL) using the conventional ABR frequency band $(150-1500 \mathrm{~Hz})$ as well as the applied five subsequent narrow frequency bands $(150-300 \mathrm{~Hz}$, $301-450 \mathrm{~Hz}, 451-600 \mathrm{~Hz}, 601-750 \mathrm{~Hz}, 751-1000 \mathrm{~Hz}$ ), illustrating the induced ringing artifact. Below the corresponding WPSS is depicted, respectively.

Note that the prior estimated NHs' ABR latencies closely follow our findings using both stimuli (Equation 7). The resulting $W_{V}$ is marked by red arrows, respectively. Furthermore, the maximum phase synchronization occurs in the downslope area of the corre- 
sponding $W_{V}$ in the averaged potential. Especially the pseudofrequency range of approximately $150-300 \mathrm{~Hz}$ (scales 40-75) seems to hold a notable part of the desired information (bottom row in Figure 1). Note that the narrowband filtered, averaged responses appear obviously more rounded and that they are additionally superimposed by the ringing artifact, but nevertheless still represent correlating components of the conventionally filtered ABR like the desired $W_{V}$ (blue lines in Figure 1). ${ }^{24}$ In contrast, the other subsequent narrowband signals do barely or not at all represent those components. This supports the assumption that the band limitation to the suggested frequency band of 150-300 Hz reduces the influence of the broadband $\mathrm{CI}$ artifact while still holding essential ABR information when further analyzing the $\mathrm{CI}$ free-field responses. However, at this point the temporal occurrences of the CI responses are still unknown. Although we further use the individual WPSS maxima as a temporal appearance indicator just as demonstrated in Figure 1, the narrow- band responses exhibit considerable ringing, making it difficult to indicate the true ABR patterns. Therefore, to prevent misinterpretation of signal components, we tested all used stimuli in four different intensities to additionally get the information of increasing $W_{V}$ maxima alongside the WPSS magnitudes and decreasing $W_{V}$ latencies to validate the expected neural behavior according to increasing stimuli intensities. $^{7}$

\section{Results}

Figure 2 depicts the grand average narrowband responses of the NH group using the chirp (left column) and click (right column) with different intensities (Figure 2A-B) as well as the intensity specific fullscale WPSS (Figure 2C-D) for the pseudofrequencies of 150-300 Hz. For the chirp stimulation responses, we

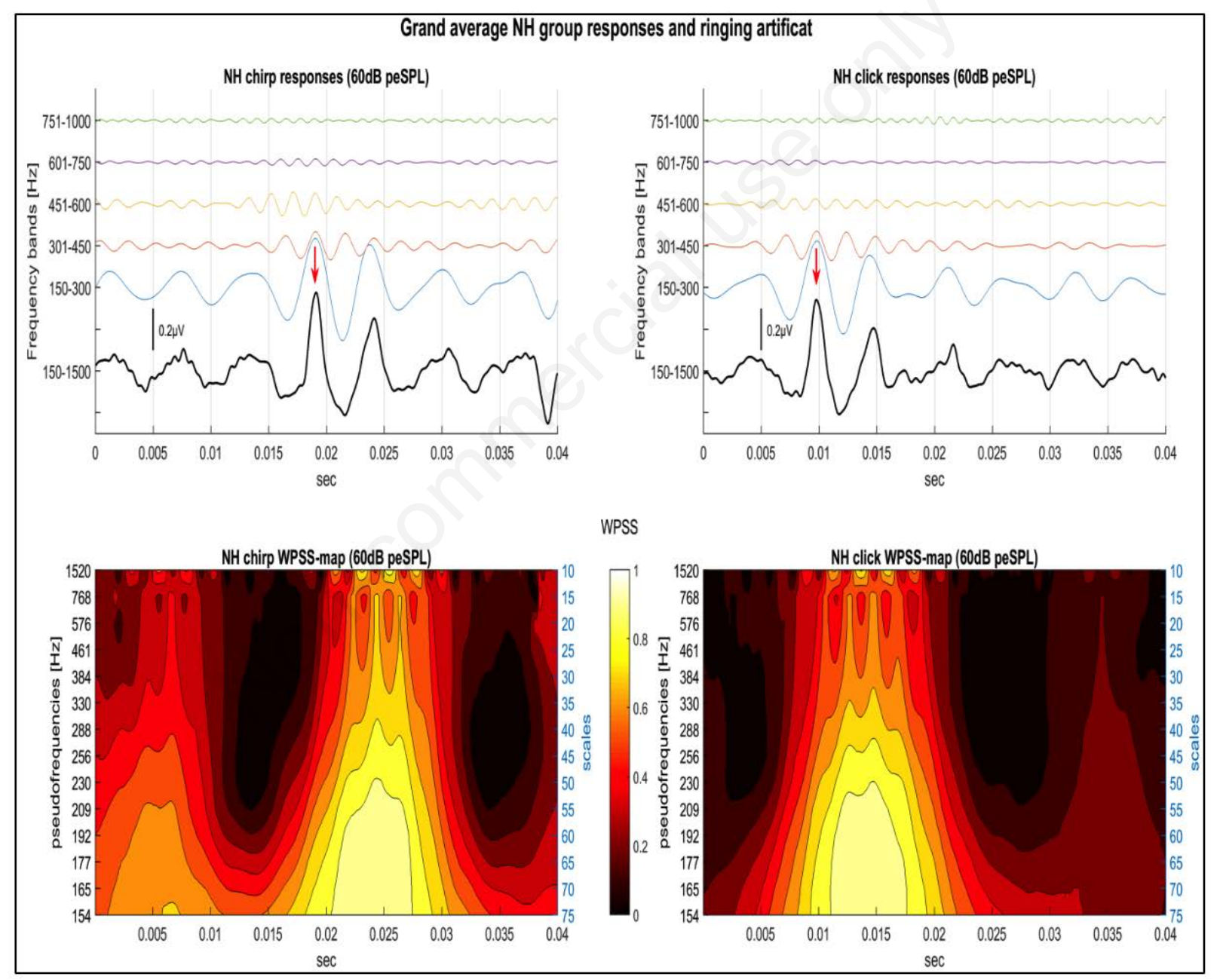

Figure 1. Grand average normal hearing (NH) group auditory brainstem evoked responses (ABR) (60 $\mathrm{dB}$ peSPL) and below the corresponding wavelet phase synchronization stability (WPSS) for the chirp (left column) and the click (right column). The conventional ABR frequency band $(150-1500 \mathrm{~Hz})$ is denoted by the black line in both plots where the $W_{V}$ is indicated by red arrows. Both plots additionally show the average responses after narrowband filtering in subsequent frequency bands demonstrating the ringing artifact caused by the filter: 150-300 Hz (blue), 301-450 Hz (orange), 451-600 Hz (yellow), 601-750 Hz (purple), and 751-1000 Hz (green). Note that the individual WPSS maxima take place in the downslope area of the corresponding $W_{V}$ in the averaged potential, especially in the pseudofrequency range of approximately $150-300 \mathrm{~Hz}$ (scales 40-75), which is one of the drawn frequency bands (blue). Further note, this average response appears more rounded but still reflects the desired features of the ABR (compare the black and blue average response especially around the temporal appearance of the individual $W_{V}$ at $\approx 18-19 \mathrm{~ms}$ (chirp) and at $\approx 10 \mathrm{~ms}(\mathrm{click})$. 
found the WPSS maxima at approximately $20 \mathrm{~ms}$ and at $11 \mathrm{~ms}$ for the click responses (denoted by the dotted vertical lines). This temporal position reflects the downslope area of the desired $W_{V}$ in the averaged potentials (maxima prior to the average WPSS, denoted by red arrows), respectively. Additionally, grand averaged WPSS-maps representing responses to an intensity of $60 \mathrm{~dB}$ peSPL are shown (Figure 2E-F), illustrating that a noteworthy regularity of oscillations taking place in the pseudofrequency range of $150-300 \mathrm{~Hz}$ (scales $40-75$ ). The ABRs using both stimuli offer distinguishable patterns in the time domain as well as in the WPSS, which increase in amplitude/magnitude and decrease in latency with increasing stimulus intensities. In the average potentials the $W_{V}$ occurs at approximately $18-20 \mathrm{~ms}$ when using chirpand at 9-10 ms when using click-stimulations, with amplitudes in the range of $0.15-0.25 \mu \mathrm{V}$. Figure 3 depicts the grand average narrowband $\mathrm{CI}$ responses, with the figure structure being analogous to that of Figure 2. For both used stimuli, the global average WPSS maxima occur later in comparison at approximately $26 \mathrm{~ms}$ for the chirp- and $17 \mathrm{~ms}$ for the click-stimulation while drifting more in the minimum-area after the desired $W_{V}$ maxima. The amplitudes are approximately ten times larger $(\approx 1-2 \mu \mathrm{V})$ than those of the $\mathrm{NH}$ group while showing a higher degree of oscillations, making it more difficult to detect the $W_{V}$. We continued with the procedure of interpreting the maxima in the average potential prior to the global temporal position of the WPSS respectively, where we found the $W_{V}$ at approximately 21-23 ms when using chirp-stimulations and at $14-15 \mathrm{~ms}$ when using click-stimulations. In comparison, the average $W_{V}$ maxima of the CI users show not quite a distinct growth pattern than those of the $\mathrm{NH}$ users. Therefore, the WPSS maxima show a clear increase in magnitude according to increasing stimulus intensities. An overview of the detected intensity and stimulus specific median values and their variances for the $W_{V}$ maxima (upper row), the $W_{V}$ latency (second row), the WPSS maxima (third row), and the WPSS latency (bottom row) for both groups is given in Figure 4. Both groups show an increase in amplitude and WPSS with both used stimuli alongside increasing stimulus intensities. However, the variances in the CI group appear to be larger than in the NH group, which is also

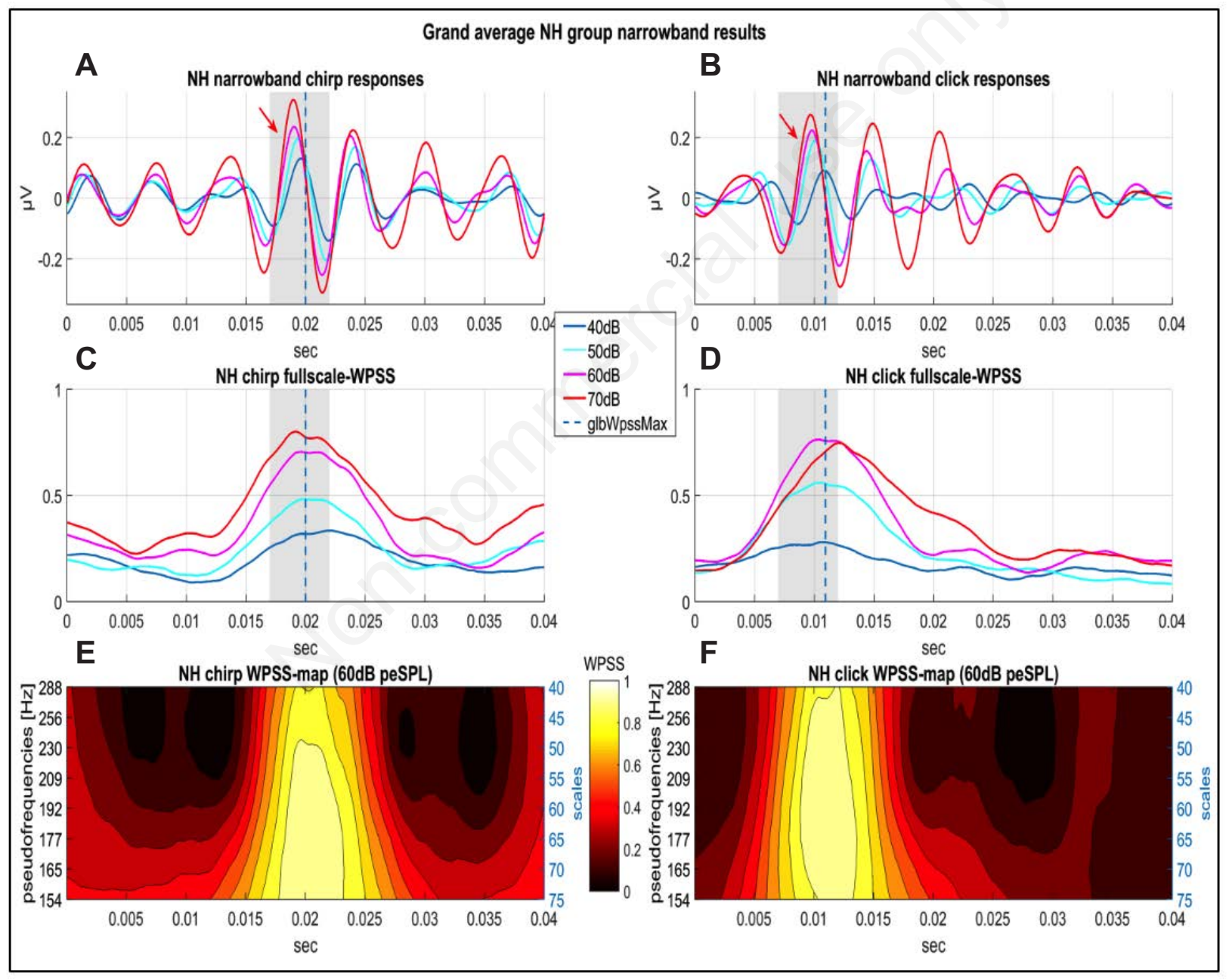

Figure 2. Grand average NH group free-field narrowband responses, where averaged temporal positions of the global WPSS maxima are denoted by vertical dotted lines at $\approx 20 \mathrm{~ms}$ (chirp) and at $\approx 11 \mathrm{~ms}$ (click). These positions are used as temporal indicators for the desired $W_{V} s$ (prior these positions). The responses for chirp (A) and click (B) show an intensity specific growing pattern in the time domain, especially prior the temporal WPSS based indicator (marked by red arrows) as well as a latency shift. A similar growing behavior is shown by the intensity specific fullscale WPSS for the chirp (C) and click (D). To improve comparability, areas of interest are highlighted by grey boxes. The intensity specific WPSS-maps, in our case those representing the grand average responses to an intensity of $60 \mathrm{~dB}$ peSPL for the chirp (E) and click (F), indicate that a noteworthy synchronization takes place in the pseudofrequency range of $150-300 \mathrm{~Hz}$ (scales 40-75). 
to a certain extent expected due to individual CI performance. Furthermore, both groups show a decrease in $W_{V}$ latency but not in the WPSS latency. Additionally, for all investigated features, we calculated the Pearson linear correlation coefficient $r_{p}$ (displayed in each corresponding legend box in Figure 4) using the median values to quantify a possible linear increase (along the $W_{V}$ and the WPSS maxima) or a decrease (along the $W_{V}$ and WPSS latency) of the detected values. Accordingly, we found positive correlations for the $W_{V}$ amplitudes and WPSS magnitudes as well as negative correlations for the $W_{V}$ latencies but no distinct correlations for the WPSS latencies for both used stimuli in both groups. Finally, we present the results of our statistical analysis in Table 2, where we first determined Friedman's non-parametric statistical ranking test (first column) to detect if there are significant differences in treatment across multiple test attempts (in our case different intensities for each used stimulus). In the conventional filtered NH group responses we found significant differences in all tested features. In narrowband filtered responses, we found significant differences in all tested features except in the WPSS latency using both stimuli in both groups and in the CI chirp latency. Additionally, we calculated the one-tailed Wilcoxon rank-sum paired difference test between all used intensities for all features (columns 2-6). In the conventionally filtered NH group responses, only single tested pairs show no significant difference. When testing the narrowband filtered $\mathrm{NH}$ group responses, we also found significant differences in the $W_{V}$ amplitudes, $W_{V}$ latencies, and WPSS magnitudes for both stimuli. However, amplitude and magnitude differences between $60 \mathrm{~dB}$ and $70 \mathrm{~dB}$ peSPL show no significant differences alongside all tested WPSS latencies. When investigating the narrowband filtered CI group responses, we found significant differences in the tested $W_{V}$ amplitudes except between $40 \mathrm{~dB}$ and $50 \mathrm{~dB}$ peSPL and the corresponding WPSS magnitudes in both used stimuli. Therefore, we found only sporadic significant differences in the CI users $W_{V}$ latency and none in the WPSS latency. To improve readability, we marked the test results with $*(\mathrm{P} \leq 0.05), * *(0.05<\mathrm{P} \leq 0.1)$, and $* * *(0.1<\mathrm{P})$.

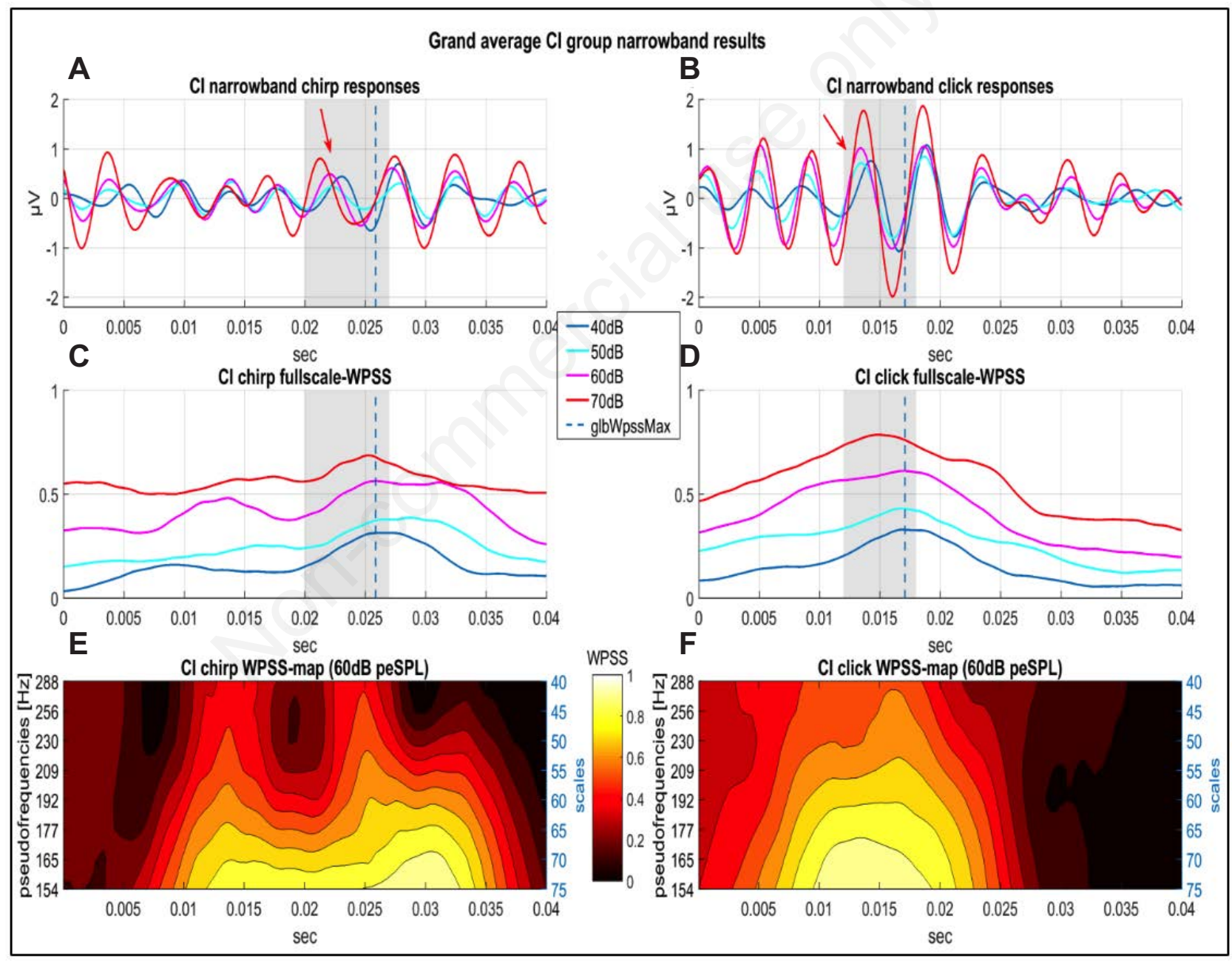

Figure 3. Grand average cochlear implant (CI) group free-field narrowband responses, where averaged temporal positions of the global WPSS maxima are denoted by vertical dotted lines at $\approx 26 \mathrm{~ms}$ (chirp) and at $\approx 17 \mathrm{~ms}$ (click). These positions are used as temporal indicators for the desired $W_{V}$ s (prior these positions). The responses for chirp (A) and click (B) suggest an intensity specific growing pattern in the time domain, especially prior the temporal WPSS based indicator (marked by red arrows) as well as a minor latency shift. A distinctly growing relation is shown by the intensity specific fullscale WPSS for the chirp (C) and click (D). To improve comparability, areas of interest are highlighted by grey boxes. The intensity specific WPSS-maps, in this case those representing the grand average responses to an intensity of $60 \mathrm{~dB}$ peSPL for the chirp $(E)$ and click $(F)$, indicate that a distinct synchronization takes place in the pseudofrequency range of $150-300 \mathrm{~Hz}$ (scales $40-75)$. 


\section{Discussion}

The grand average of the free-field ABRs for the NH group for both stimuli ( $60 \mathrm{~dB}$ peSPL) when using conventional filter boundaries $(150-1500 \mathrm{~Hz})^{7}$ are shown in Figure 1 (black lines), where common ABR patterns and their $W_{V}$ s are clearly identifiable (marked by red arrows). Note that the prior estimated latencies by Equation 7 resemble those of the desired components, demonstrating the general feasibility of free-field ABR measurements in NHs, additionally supported by statistical results (Table 2). However, when testing this in CI users, responses are affected by the prominent broadband $\mathrm{CI}$ artifact ${ }^{14-18}$ making it almost impossible to use conventional filter boundaries alongside an artifact filter with meaningful threshold. Actually, when using the conventional approach hardly any single trials could be collected in any CI user. When totally excluding artifact filtering only one single CI user showed an interpretable ABR pattern (not shown). Therefore, based on the work of Luu and Tucker ${ }^{27}$ and Yeung et al., ${ }^{28}$ we searched for the frequency window that holds for the most part the desired exogenously driven synchronizing oscillations causing the prominent $W_{V}$ in the average potential. Using the WPSS as a supporting indicator, we found the peak frequency within the range of 150-300 Hz (bottom row in Figure 1), where a noteworthy synchronization takes place. Note that a similar narrowband ABR frequency window was successfully investigated by Corona-Strauss et al. ${ }^{24}$ Consequently, this narrowband is used in both groups to gain comparable results while additionally excluding parts of the $\mathrm{CI}$ artifact in the subsequent analysis. However, when narrowband filtering evoked potentials those might get distorted by the so called ringing artifact. ${ }^{27,28}$ Since it is known that the magnitude of the ringing artifact will depend on the size of the original peak (blue line in Figure 1) in the average pattern, we tested four different intensities in both stimuli to additionally investigate possible changes in the intensity specific $W_{V}$. This step is necessary because it offers additional evidence if the correct peak (the desired response) is found. This becomes even more obvious when considering that so far the temporal appearance of free-field evoked CI $W_{V} \mathrm{~S}$ is not known. Here, we also adapt our findings in the $\mathrm{NH}$ group to the CI group, where the maximum phase synchronization appears in the local minimum after the $W_{V}$ in the average potential (Figure 1), what induces the method of interpreting the very first

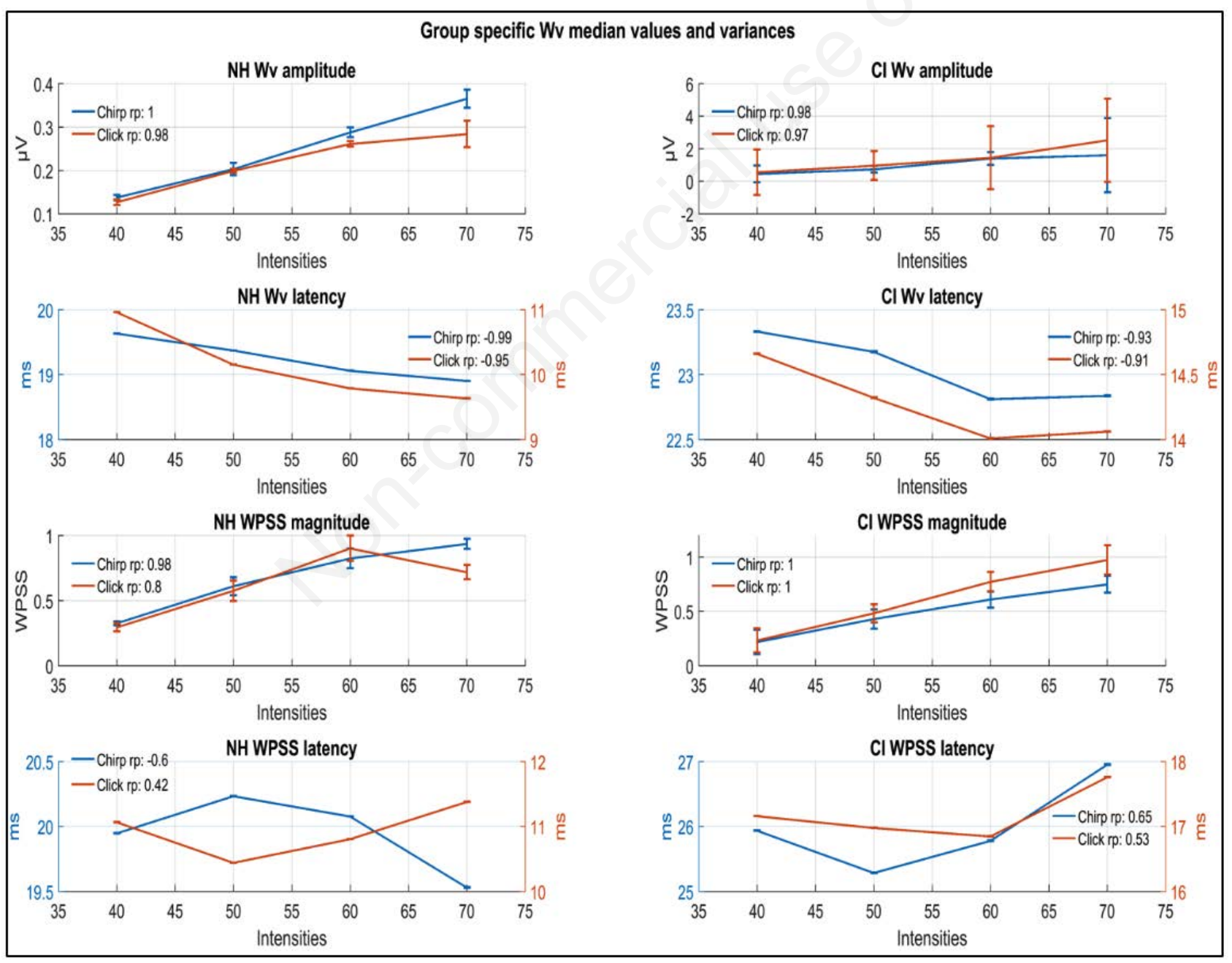

Figure 4. Overview of the free-field narrowband response features for: the NH group, on the left, and the CI group, on the right, representing the median values and the corresponding variances as error bars for the chirp (blue) and click (red). Additionally all plots show the calculated Pearson linear coefficient $\mathbf{r}_{\mathrm{p}}$ within the legend box. Top row: Grand average $W_{V}$ amplitudes and variances. Second row: Grand average $W_{V}$ latencies and variances. Third row: Grand average WPSS magnitudes and variances. Bottom row: Grand average WPSS latencies and variances. 
maximum prior the temporal WPSS maximum as the desired neural correlate of the $W_{V}$. Figure $2(\mathrm{NH})$ and Figure $3(\mathrm{CI})$ show the group specific grand average results for both used stimuli, respectively. Note the mentioned temporal dependencies between the global WPSS maxima (denoted by the vertical dotted lines) and the leading maxima in the average intensity specific potentials, where the NHs show temporal WPSS maxima at $\approx 20 \mathrm{~ms}$ when using chirp-stimulations and at $\approx 11 \mathrm{~ms}$ ms when using click-stimulations. In contrast, CI users show those maxima at $\approx 26 \mathrm{~ms}$ when using chirp-stimulations and at $\approx 17 \mathrm{~ms}$ when using click-stimulations. This shows a consistent temporal lag for both stimuli of $\approx 6$ $\mathrm{ms}$ according to the obtained WPSS maxima and of $\approx 3-4 \mathrm{~ms}$ according to the obtained $W_{V}$ maxima between both groups. Consequently, this has implications for the current research field of ITDs and binaural interaction ${ }^{4-6,12,13}$ since interaural lags exceeding 600-1000 $\mu$ s will arguably prohibit meaningful integration of binaurally presented information at the brainstem level. When investigating the neural behavior according to increasing stimulus intensities ${ }^{7}$ the NHs' distinctly show the expected $W_{V}$ amplitude growth as well as the expected latency shifting in both used stimuli (Figure 2A-B). This is also partially visible in the corresponding WPSS (Figure 2C-D). By contrast, this neural behavior is only barely visible in the CI users' grand average responses (Figure 3AB) but holds when investigating the corresponding WPSS (Figure
3C-D). For a better comparison, the identified $W_{V}$ features $\left(W_{V}\right.$ amplitudes, WPSS magnitudes, and corresponding latencies) are summarized in Figure 4, showing the grand average median values as well as their respective variances for both groups, respectively. In general, an increase in amplitudes and magnitudes alongside a decrease in $W_{V}$ latencies is observable for both groups and stimuli with increasing stimulus intensities. An observation that needs to be discussed is the around ten times increased response amplitude in CI users. A possible explanation is based on results of eABR measurements, ${ }^{8-10}$ where $W_{V}$ amplitudes are in fact in the range of those of conventional ABRs. The major difference is that we especially aimed for a use of the whole electrode array in our experiment (instead of using single isolated electrodes like in conventional eABR measurements) by using broadband stimuli (chirp and click) potentially leading to a summation of eABRs in the range of the number of active implant electrodes $(>10)$. Although the implant electrodes do not fire simultaneously, the temporal lag between two successively firing electrodes can be considered negligible, supporting the idea of a summation of eABRs leading to increased $W_{V}$ amplitudes in CI users when leaving their complete electrode array active during free-field stimulation. Note that these findings are not detectable in conventional eABR measurements. The validity of this hypothesis needs further investigations, however, we emphasize that there exist no alternatives to employing

Table 2. Significance table illustrating the statistical analysis with Friedman's ranking tests (pFried) and the one-tailed Wilcoxon paired difference tests (pIntensity\# $1 \rightarrow$ Intensity $\# 2) .{ }^{*}(\mathrm{P} \leq 0.05),{ }^{* *}(0.05<\mathrm{P} \leq 0.1)$, and ${ }^{* * *}(0.1<\mathrm{P})$.

\begin{tabular}{|c|c|c|c|c|c|c|c|}
\hline Tested features & pFried & p40 $\rightarrow 50$ & $\mathrm{p} 40 \rightarrow 60$ & $\mathrm{p} 40->70$ & $\mathrm{p} 50 \rightarrow 60$ & $\mathrm{p} 50 \rightarrow 70$ & $\mathrm{p} 60 \rightarrow 70$ \\
\hline \multicolumn{8}{|c|}{ NH group conventional frequency window $(150-1500 \mathrm{~Hz})$} \\
\hline Chirp amplitude & $0.00002 *$ & $0.00073^{*}$ & $0.00024 *$ & $0.00024^{*}$ & $0.01343^{*}$ & $0.00244^{*}$ & $0.00806^{*}$ \\
\hline Chirp latency & $0^{*}$ & $0.01563^{*}$ & $0.00049 *$ & $0.00024^{*}$ & $0.00195^{*}$ & $0.00024 *$ & $0.01636^{*}$ \\
\hline Chirp WPSS magnitude & $0.00001^{*}$ & $0.00342^{*}$ & $0.00024^{*}$ & $0.00024^{*}$ & $0.00073^{*}$ & $0.0061^{*}$ & $0.08813^{* *}$ \\
\hline Chirp WPSS latency & $0.00063^{*}$ & $0.04028^{*}$ & $0.01123^{*}$ & $0.00098^{*}$ & $0.01904^{*}$ & $0.00146^{*}$ & $0.0293^{*}$ \\
\hline Click amplitude & $0.00173^{*}$ & $0.00171^{*}$ & $0.00024^{*}$ & $0.0061^{*}$ & $0.03198 *$ & $0.1167^{* * *}$ & $0.48486 * * *$ \\
\hline Click latency & $0.00004^{*}$ & $0.02539 *$ & $0.00269 *$ & $0.00146^{*}$ & $0.00098^{*}$ & $0.00024^{*}$ & $0.00073^{*}$ \\
\hline Click WPSS magnitude & $0.00011^{*}$ & $0.00049 *$ & $0.00024^{*}$ & $0.00024^{*}$ & 0.03198* & $0.10181^{* * *}$ & $0.1167^{* * *}$ \\
\hline Click WPSS latency & $0.0007^{*}$ & $0.00293^{*}$ & $0.00293^{*}$ & $0.00049^{*}$ & $0.26538^{* * *}$ & $0.00391^{*}$ & $0.00195^{*}$ \\
\hline \multicolumn{8}{|c|}{ NH group narrowband frequency window (150-300 Hz) } \\
\hline Chirp amplitude & $0.00002 *$ & $0.00806^{*}$ & $0.00122 *$ & $0.00024 *$ & $0.04614 *$ & $0.00122 *$ & $0.00806^{*}$ \\
\hline Chirp latency & $0.00004 *$ & $0.00146^{*}$ & $0.00024 *$ & $0.00049 *$ & $0.03027^{*}$ & $0.02051^{*}$ & $0.10938 * * *$ \\
\hline Chirp WPSS magnitude & $0.00002 *$ & $0.00806^{*}$ & $0.00024 *$ & $0.00024 *$ & $0.0105^{*}$ & $0.00806 *$ & $0.08813^{* *}$ \\
\hline Chirp WPSS latency & $0.09789^{* *}$ & $0.06177^{* *}$ & $0.05713^{* *}$ & $0.37378^{* * *}$ & $0.29102^{* * *}$ & $0.08813^{* *}$ & $0.10547^{* * *}$ \\
\hline Click amplitude & $0.00033^{*}$ & $0.01343^{*}$ & $0.00049^{*}$ & $0.00244^{*}$ & $0.08813^{* *}$ & $0.0105^{*}$ & $0.1167^{* * *}$ \\
\hline Click latency & $0^{*}$ & $0.00122^{*}$ & $0.00024^{*}$ & $0.00024 *$ & $0.00024^{*}$ & $0.00024 *$ & $0.00293^{*}$ \\
\hline Click WPSS magnitude & $0.00007^{*}$ & $0.00073^{*}$ & $0.00024^{*}$ & $0.00024 *$ & $0.0061^{*}$ & $0.04614 *$ & $0.33862 * * *$ \\
\hline Click WPSS latency & $0.2301^{* * *}$ & $0.38232^{* * *}$ & $0.16602^{* * *}$ & $0.22876^{* * *}$ & $0.49121^{* * *}$ & $0.13306^{* * *}$ & $0.146^{* * *}$ \\
\hline \multicolumn{8}{|c|}{ CI group narrowband frequency window $(150-300 \mathrm{~Hz})$} \\
\hline Chirp amplitude & $0.01346^{*}$ & $0.1167^{* * *}$ & $0.00464^{*}$ & $0.00806^{*}$ & $0.00806^{*}$ & $0.0105^{*}$ & $0.03198 *$ \\
\hline Chirp latency & $0.45198^{* * *}$ & $0.09033^{* *}$ & $0.04614^{*}$ & $0.06738^{* *}$ & $0.24023^{* * *}$ & $0.2832^{* * *}$ & $0.4248^{* * *}$ \\
\hline Chirp WPSS magnitude & $0.00085^{*}$ & $0.08813^{* *}$ & $0.00122 *$ & $0.0105^{*}$ & $0.00024^{*}$ & $0.03857^{*}$ & $0.25928^{* * *}$ \\
\hline Chirp WPSS latency & $0.43678^{* * *}$ & $0.37427^{* * *}$ & $0.3042^{* * *}$ & $0.15552^{* * *}$ & $0.13428^{* * *}$ & $0.21191^{* * *}$ & $0.22607^{* * *}$ \\
\hline Click amplitude & $0.00007^{*}$ & $0.05493^{* *}$ & $0.00244^{*}$ & $0.00073^{*}$ & $0.00244 *$ & $0.00122 *$ & $0.01709 *$ \\
\hline Click latency & $0.02964 *$ & $0.02002^{*}$ & $0.05688^{* *}$ & $0.00806^{*}$ & $0.17188^{* * *}$ & $0.07275^{* *}$ & $0.32715^{* * *}$ \\
\hline Click WPSS magnitude & $0.00026^{*}$ & $0.01343^{*}$ & $0.00342 *$ & $0.00244^{*}$ & $0.00171^{*}$ & $0.02124 *$ & $0.03857^{*}$ \\
\hline Click WPSS latency & $0.23084^{* * *}$ & $0.40283^{* * *}$ & $0.49341^{* * *}$ & $0.19531^{* * *}$ & $0.13306^{* * *}$ & $0.13721^{* * *}$ & $0.14624 * * *$ \\
\hline
\end{tabular}


the whole electrode array and associated preprocessing chain (microphone, sound processor and filter bank) in obtaining evoked responses closely representing the users' daily-life listening situations. Using statistical analysis (Friedman and Wilcoxon tests) (Table 2), we found significant paired differences between the used intensities in nearly all conventionally filtered features of the $\mathrm{NH}$ group. We also found significant differences in nearly all narrowband filtered $W_{V}$ amplitudes in both stimuli and groups, indicating that differences of approximately $10 \mathrm{~dB}$ peSPL are for the most part distinguishable using the neural correlate. However, intensity steps of $20 \mathrm{~dB}$ peSPL exhibit significant increases across all compared intensity pairs in both groups. Comparable results are observable for the WPSS magnitude in both stimuli and groups, where $10 \mathrm{~dB}$ steps do not exhibit significant increases in the neural correlate, while $20 \mathrm{~dB}$ steps do. By contrast, this behavior changes when investigating the latencies of narrowband filtered responses. We could not observe significant differences in any WPSS latency in both stimuli and groups. While we found significant differences in narrowband filtered NHs' $W_{V}$ latencies in almost every condition, results were not significant for the CI users, although Figure 4 (second row/column) indicates a similar behavior. Note that the calculated Pearson linear correlation coefficients also support these findings. However, there are two possible explanations. On the one hand this might be affected by the narrowband filtering itself, excluding higher frequencies, what potentially reduces temporal resolution. These assumptions are based on the statistical findings, where WPSS latencies are distinguishable when using the conventional frequency window but not when using the narrowband frequency window in the $\mathrm{NH}$ group, where the difference in treatment is narrowband filtering. On the other hand temporal resolution of scale-average WPSS (fullscale WPSS) is bounded by the time-frequency uncertainty of the lowest incorporated scale, thus potential significant changes in the peak locations may be masked by temporal smearing. To sum up, the interpretation of conventionally filtered CI users' free-field responses alongside meaningful artifact filtering when leaving the complete electrode array active is practically infeasible. Although signal quality and differentiability get altered when those responses being narrowband filtered, visual (Figures 2-4) alongside statistical (Table 2) analysis of those responses do in fact grant unique accessibility for interpretation of CI users' true-to-life responses at brainstem level.

\section{Conclusions}

In this study, we introduce a novel method to measure and analyze narrowband filtered neural correlates of free-field ABRs in CI users that does not bypass CI preprocessing, making an investigation of evoked responses to real-world stimuli and a comparison to those of NHs feasible. This approach is unique and shows its benefits in comparison to eABR measurements, which bypass CI preprocessing altogether and only concentrate on single isolated electrodes, which does not reflect CI users' true-to-life listening situation. It could objectively support CI fitting procedures and future studies aiming at optimal binaural interaction and fusion, where the neural processing latencies and ITDs are fundamental. Therefore, more participants are needed to further improve the statistical power of the obtained results. Another potentially beneficial refinement of the presented paradigm would be the incorporation of the individual sensation levels rather than objective intensities, most likely leading to smaller variances, since it would better reflect the individuals' sensory perception. We are going to address these points in future studies.

\section{References}

1. Zeng F. Trends in cochlear implants. Trends Amplif 2004;8:134.

2. Bregman A, Rudnicky A. Auditory segregation: Stream or no streams. J Exp Psychol Human 1975;1:263-7.

3. Bahmer A, Polak M, Baumann U. Recording of electrically evoked auditory brainstem responses after electrical stimulation with biphasic, triphasic and precision triphasic pulses. Hearing Res 2010;259:75-85.

4. Bures Z. The stochastic properties of input spike trains control neuronal arithmetic. Biol Cybern 2012;106:111-22.

5. Schebsdat E, Hessel H, Seidler H, Strauss DJ. Detection of binaural interaction in free-field evoked auditory brainstem responses by time-scale representations. Conf Proc IEEE Eng Med Biol Soc 2016; Orlando, FL, USA.

6. Agmon-Snir H, Carr C, Rinzel J . The role of dendrites in auditory coincidence detection. Nature 1998;393:168-272.

7. Corona-Strauss F. On the fast detection of auditory brainstem responses: A novelty detection paradigm. $\mathrm{PhD}$ thesis, Universität des Saarlandes, Saarbruecken, Germany; 2009.

8. Thai-Van H, Cozma S, Boutitie F, et al .The pattern of auditory brainstem response wave $\mathrm{v}$ maturation in cochlear-implanted children. Clin Neurophysiol 2007;118:676-89.

9. Klauke I, Kohl MC, Hannemann R, et al. Impact of monaural frequency compression on binaural fusion at the brainstem level. Conf Proc IEEE Eng Med Biol Soc 2015; Milan, Italy.

10. Firszt J, Chambers R, Kraus N, Reeder R. Neurophysiology of cochlear implant users: Effects of stimulus current level and electrode site on the electrical ABR, MLR, and N1-P2 response. Ear Hearing 2002;23:502-15.

11. Pichora-Fuller M, Singh G. Effects of age on auditory and cognitive processing: implications for hearing aid fitting and audiologic rehabilitation. Trends Amplif 2006;10:29-59.

12. Purdy S, Gardner-Berry K, Sharma M, et al. Electrophysiological measures of binaural interaction in cochlear implantees. Int Congr Ser 2004;1273:40-3.

13. Grau-Serrat V, Carr C, Simon J. Modeling coincidence detection in nucleus laminaris. Biol Cybern 2003;89:388-96.

14. Sinkiewicz D, Friesen L, Ghoraani B. Analysis of cochlear implant artifact removal techniques using the continuous wavelet transform. Conf Proc IEEE Enf Med Biol Soc 2014; Chicago, IL, USA.

15. McLaughlin M, Lopez Valdes A, Reilly R, Zeng F. Cochlear implant artifact attenuation in late auditory evoked potentials: a single channel approach. Hear Res 2013;302:84-95.

16. Friesen L, Picton T. A method for removing cohlear implant artifact. Hear Res 2010;259:95-106.

17. Gilley P, Sharma A, Dorman M, et al. Minimization of cochlear implant stimulus artifact in cortical auditory evoked potentials. Clin Neurophysiol 2006;117:1772-82.

18. Atcherson S, Damji Z, Upson S. Applying a subtraction technique to minimize cochlear implant artifact with soundfield and direct audio input stimulations. Cochlear Implants Int 2011;12:234-7.

19. Brown C, Abbas PJ. Electrically evoked whole-nerve action potentials: parametric data from the cat. J Acoust Soc Am 1990;88:2205-10.

20. Brown CJ, Abbas PJ, Gantz B. Electrically evoked wholenerve action potentials: data from human cochlear implant users. J Acoust Soc Am 1990;88:1385-91.

21. Dillier N, Lai W, Almqvist B, et al. Measurement of the electrically evoked compound action potential via a neural response telemetry system. Ann Otol Rhinol Laryngol 
2002;11:407-14.

22. Fobel O, Dau T. Searching for the optimal stimulus elicting auditory brainstem responses in humans. J Acoust Soc Am 2004; 116:2213-22.

23. Corona-Strauss F, Delb W, Bloching M, Strauss DJ. Ultra-fast quantification of hearing loss by neural synchronization stabilities of auditory evoked brainstem activity. Conf IEEE Eng Med Biol Soc 2007; Minneapolis, Minnesota, USA.

24. Corona-Strauss F, Delb W, Schick B, Strauss DJ. Phase stability analysis of chirp evoked auditory brainstem responses by gabor frame operators. IEEE Trans Neural Syst Rehabil Eng 2009;17:530-6.

25. Carmona R, Hwang WL, Torresani B. Practical time-frequency analysis: Gabor wavelet transforms wit an implementation $\mathrm{S}$. San Diego, CA: Academic Press; 1998.

26. Strauss DJ, Corona-Strauss F, Bernarding C, et al. Electrophysiological correlates of listening effort: Neurodynamical modeling and measurement. Cogn Neurodyn 2010;4:119-31.

27. Luu P, Tucker DM. Regulating action: Alternating activation of midline frontal and motor cortical networks. Clin Neurophysiol 2001;112:1295-306.

28. Yeung N, Bogazc R, Holroyd CB, Cohen JD. Detection of synchronized oscillations in the electroencephalogram: An evaluation of methods. Psychology 2004;41:822-32. 